\title{
EL REINO LUPAQA: ARTICULACIÓN ENTRE TIERRAS ALTAS Y BAJAS
}

\author{
THE LUPAQA KINDOM: AN ARTICULATION BETWEEN \\ HIGH LANDS AND LOW LANDS
}

\author{
Estela Cristina Salles ${ }^{*}$ y Héctor Omar Noejovich**
}

\begin{abstract}
Este reino ubicado en la cuenca del lago Titicaca, de origen aymara, fue un centro que relacionó desde el altiplano asentamientos tanto en zonas subtropicales de altura como costeñas.

El sistema, que en quechua se refiere a los mitmaqkuna, tuvo una significación muy alta en la circulación de bienes, tanto prehispánica como colonial.
\end{abstract}

Palabras claves: Titicaca, altiplano, aymara, mitmaqkuna.

This kingdom has been located on Titicaca basin with Aymara roots. It worked as intermediary among the Andean high plateau, subtropical and coastal areas.

The system, named in Quechua as mitmaqkuna one, had an important role for goods circulation either in pre-colonial time as well as in colonial ones.

Key words: Titicaca, plateau, aymara, mitmaqkuna.

\section{Presentación}

A manera de introducción comencemos con la ubicación actual del lago Titicaca, centro de la etnia que constituye el objeto de esta, y las características climáticas cuyos cambios en el tiempo tuvieron influencia en los patrones de asentamiento y las estrategias socioeconómicas, incluso hasta el presente (Mapa 1) ${ }^{1}$.

El asiento principal de la etnia Lupaqa era la margen occidental del lago y se conocía como la provincia de Chucuito nombre vigente en la actualidad; ubicada en el departamento de Puno, República del Perú, aproximadamente entre los $16^{\circ}$ de latitud Sur y $70^{\circ}$ de longitud Oeste; la altitud está comprendida entre los 3.800 m.s.m. y $\operatorname{los} 3.900$ m.s.m.

Se conservan asimismo las seis cabeceras restantes de las siete que formaban el asentamiento: Acora, Ilave (a orillas del río homónimo), Juli, Pomata, Yunguyo y Zepita; estos dos últimos a orillas de la laguna de Huiñamarca, separada del lago por los estrechos de Tiquina que separan la península de Copacabana del territorio boliviano.

La zona es principalmente ganadera con cierto tipo de agricultura -papa, olluco y otros

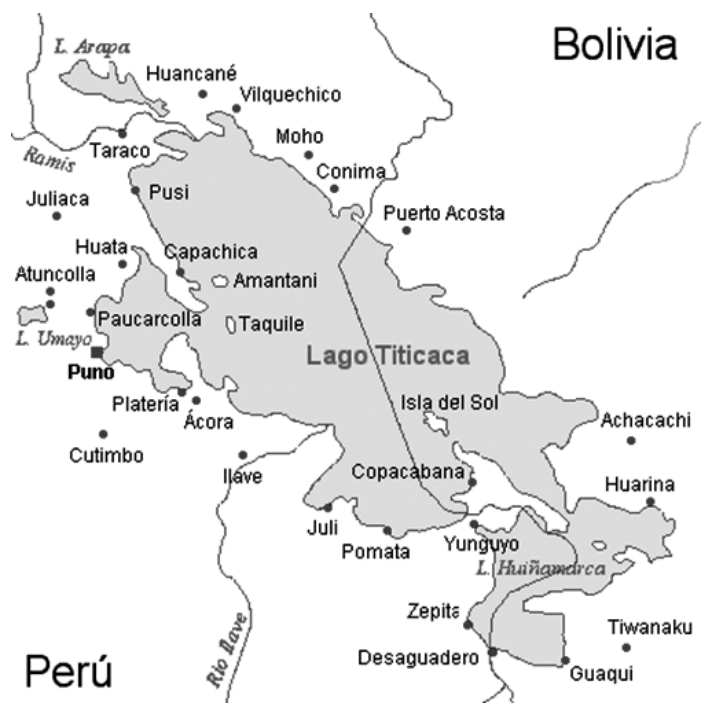

Mapa 1. Ver nota 1.

tubérculos- $\mathrm{y}$ sus precipitaciones pluviales son del orden de los $800 \mathrm{~mm}$ al año durante la estación de verano -diciembre-abril-; la temperatura media anual es de $8{ }^{\circ} \mathrm{C}$, aproximadamente, con máximas de $14{ }^{\circ} \mathrm{C}$ y mínimas de $2{ }^{\circ} \mathrm{C}$.

Una de las características de la zona aledaña es la inundación por variaciones del nivel del lago, que

\footnotetext{
* Universidad Nacional de Luján, Buenos Aires, Argentina. Correo electrónico: ecsalles@ gmail.com

** Pontificia Universidad Católica del Perú, Lima, Perú. Correo electrónico: hnoejov@ pucp.edu.pe
} 
dio origen a la agricultura en camellones o waruwaru, consistente en formar parcelas elevadas y donde se asientan las sementeras para los cultivos; tecnología prehispánica pero de utilización también actual.

Adicionalmente hay que remarcar que, especialmente en la parte occidental de los Andes, las pendientes son abruptas, generando como mínimo tres regiones: la Puna -arriba de 3500 m.s.m.-, la Quechua -entre 2000/3, 500 m.s.m.- y la Yunga debajo de los -2000 m.s.m- en distancias relativamente cortas.

\section{Los "horizontes" arqueológicos}

Aunque el modelo es materia de discusión por parte de la antropología y la etnohistoria, resulta útil para orientar al lector que no frecuenta esta área, donde las disciplinas se superponen con sus respectivas hermenéuticas; en nuestro caso esa discusión es relevante a los fines expositivos.

Las variaciones de la periodización de esos "horizontes" está en función de los hallazgos de construcciones, cerámicas y sistemas agrícolas; estos últimos indicadores de las variaciones climáticas, que explicarían las migraciones o invasiones.

El caso de las construcciones, especialmente adoratorios -también denominados templos- y chullpas -monumentos funerarios-; para el caso de la agricultura, el sistema de camellones, que mencionáramos y las qochas, estanques formados para recolectar agua de las lluvias o canales desde los ríos que forman parte de la cuenca endorreica del Titicaca.

El Cuadro $1^{2}$ permite ubicar los "horizontes", con sus respectivas correspondencias en el área, que se completa con el siguiente.

Cuadro 1. Los "Horizontes" en la cuenca del Titicaca.

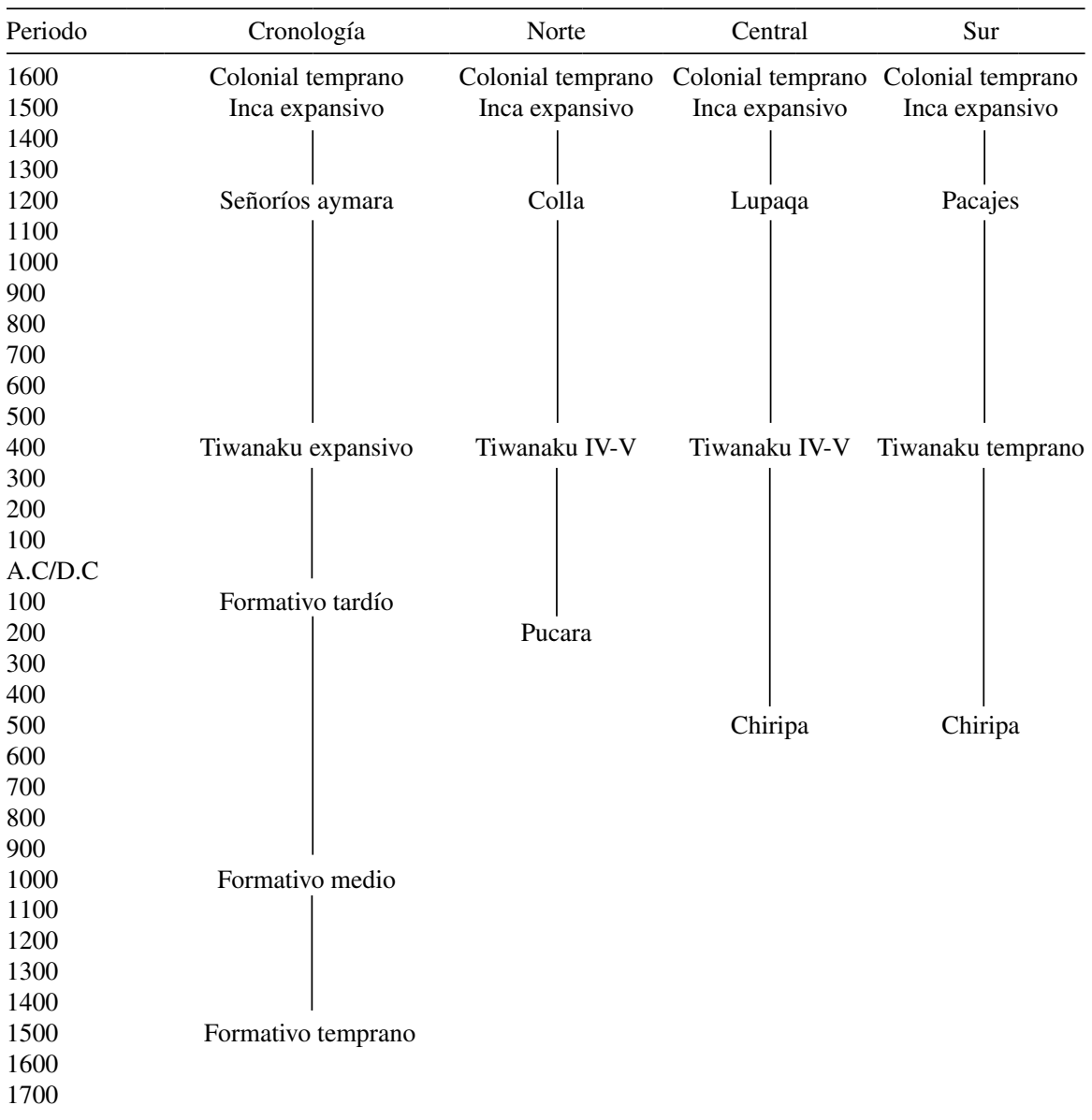

Fuente: Ver nota 2. 


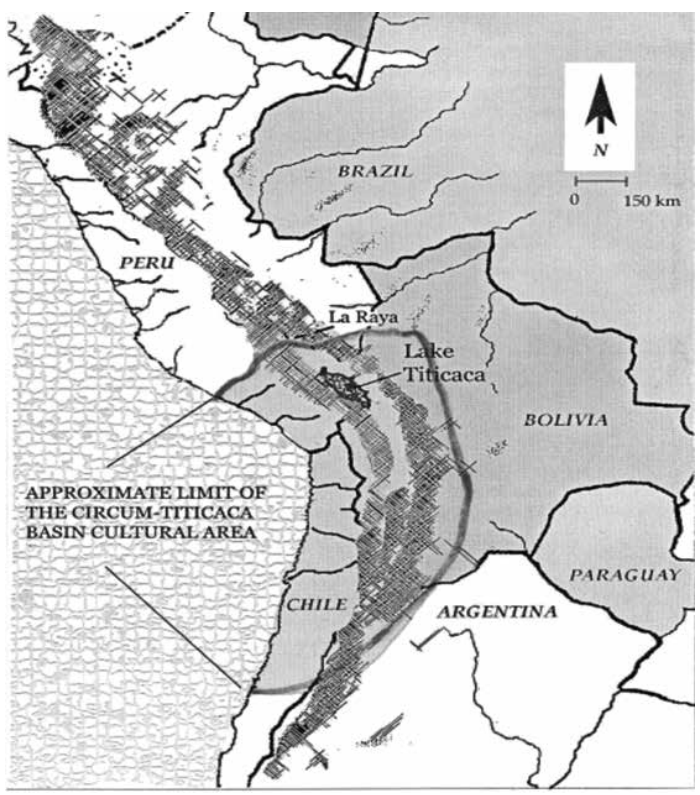

Mapa 2. Ver nota 3.

El área resaltada corresponde a la zona de influencia de las culturas de la cuenca del Titicaca (Mapa 2) ${ }^{3}$, de importancia para ir formando unas ideas acerca de la orientación occidental seguidas por esas culturas en materia de obtención de recursos e intercambios.

Queda marcada en términos geopolíticos y geoeconómicos la evolución de las culturas preincaicas en el área, estableciendo una diferencia con las orientaciones de la etnografía argentina, que hace hincapié en las formaciones al sur del Titicaca, como se aprecia en el Mapa $3^{4}$.

Regresando al área del Titicaca, los hallazgos arqueológicos indican la cultura Pucará, hacia el norte y la Chiripa hacia el sur, incluyendo el área que, posteriormente, será el espacio Lupaqa, apareciendo luego la civilización del Tiwanaku, la misma que, según Conlee, Dulanto, Mackey y Stanish, se constituyó en poder regional hacia 600 de nuestra era, con un pico en 900 y una declinación a partir de 1000; Tarragó , por su parte, fecha su hegemonía entre los siglos V y XI, en tanto Muñoz Ovalle ubica la influencia del Tiwanaku en el valle de Azapa a partir del 300.

En la arqueología peruana se reconoce el aporte de Tiwanaku en la cultura Wari, ubicada en Ayacucho, y que se extendió hacia la costa central, Lumbreras incluso se refiere a una posible invasión de la cultura altiplánica; la cronología dada por

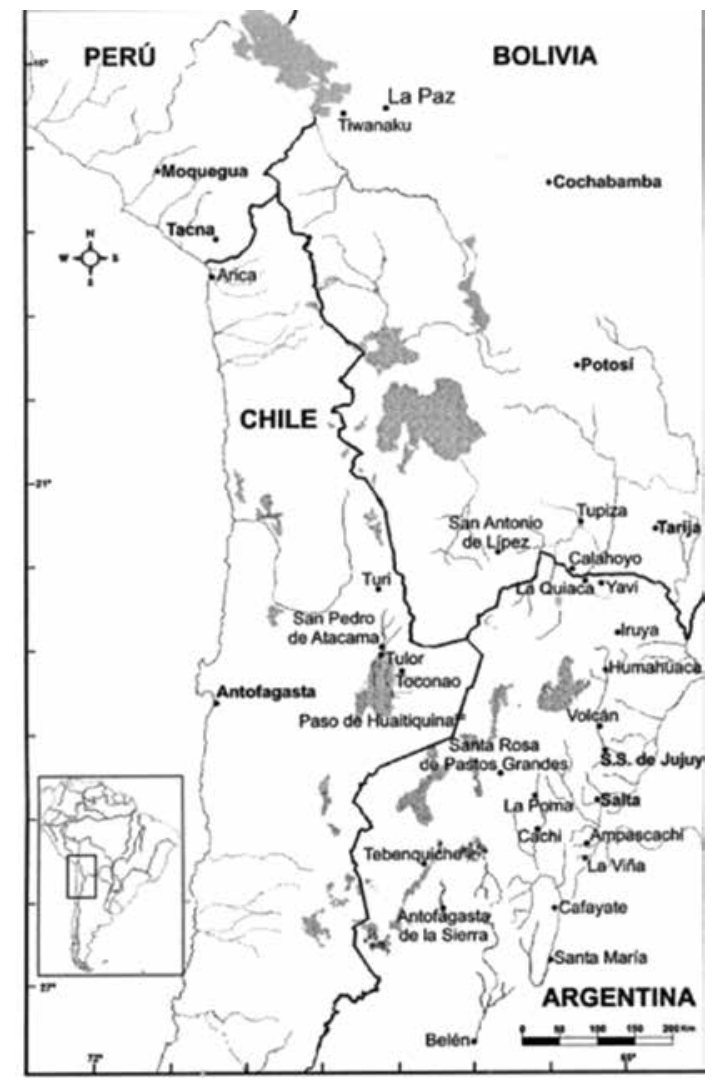

Mapa 3. Ver nota 4.

este autor, del siglo VI al siglo XI, concuerda con el periodo indicado para Tiwanaku.

Otro debate se abre sobre las causas de la caída de esa civilización; frente a las tesis de Kolata respecto de problemas climáticos -que desarrollamos en la siguiente sección-y la de Dittmar, acerca de una "invasión aymara", que no son excluyentes; veamos la de esta última.

Sobre la base antropológica que suministra la etnogénesis, la autora atribuye el origen aymara a tres movimientos: de la vertiente oriental del Titicaca, del noroeste argentino y del sur peruano; entra en el terreno de las posibilidades, pero el caso concreto generalmente aceptado es la formación de señoríos aymaras a partir del siglo XIII.

$\mathrm{Al}$ respecto Lumbreras ${ }^{5}$ señala:

"En el altiplano del lago Titicaca, la decadencia de Tiwanaku significó la emergencia de todo el contorno del inmenso lago, de una serie de reinos independientes y rivales; todos ellos 
sin embargo ligados culturalmente por muchos aspectos comunes, de modo tal que es posible hablar de una cultura regional con algunas variaciones locales, que quizá corresponden a las varias unidades políticas o étnicas que existían en el momento en que la zona fue incorporada al Imperio de los Incas".

El Mapa $4^{6}$ nos proporciona la ubicación de esos señoríos, que ha servido de base para la historiografía colonial.

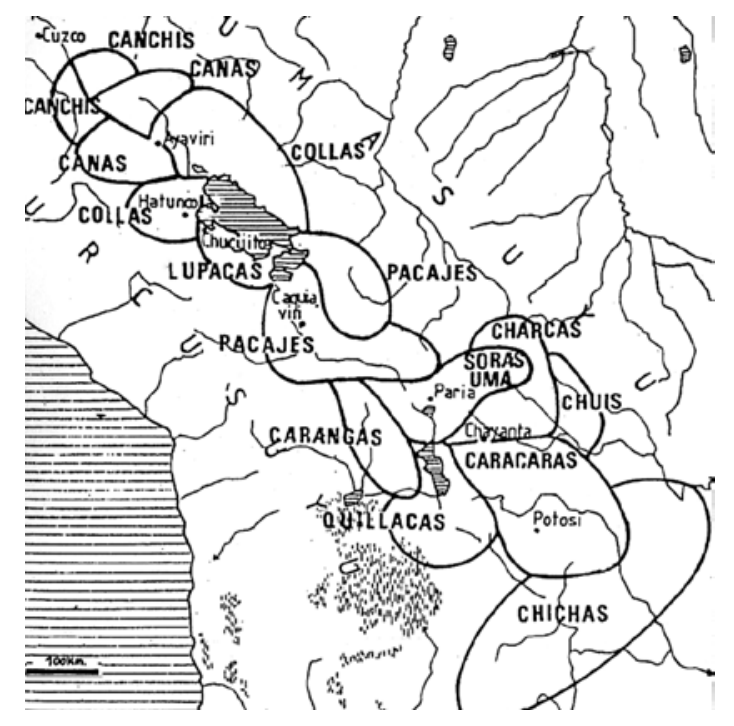

Mapa 4. Ver nota 6.

Bajo esos términos, las articulaciones de las culturas al norte de Tiwanaku, a partir de Pacajes, fueron más intensas hacia el océano Pacífico, mientras las otras, como los Caracaras y vecinos se orientaron al Este; esto es interesante remarcarlo, porque tanto la historiografía argentina como boliviana hace mayor hincapié en el hoy llamado Oriente boliviano ${ }^{7}$.

\section{El clima y la reestructuración de las articulaciones regionales}

Según $\mathrm{Kolata}^{8}$, hacia el año 1000 , estudios sobre los suelos indican una disminución de la capa de hielo, como consecuencia de un proceso de calentamiento global y disminución del régimen de lluvias, seguido de una secuela de sequías.
En el altiplano ${ }^{9}$, especialmente en la región de Hatuncolla -ver Mapa 1-, ello afectó el sistema de qochas, lagunas formadas por canales provenientes de los ríos o por las lluvias, sistema que aparentemente fue difundido por la cultura Pucará, que se extendía hacia Ayaviri, marcando la extensión de la cultura del Titicaca antes de La Raya ${ }^{10}$.

Este sistema se conecta con los camellones o waruwaru, que protegen los cultivos de las heladas y las inundaciones, bajo el principio que aire caliente desaloja al aire frío, como podemos observar el siguiente diagrama (Cuadro 2).

Cuadro 2. Croquis del camellón.

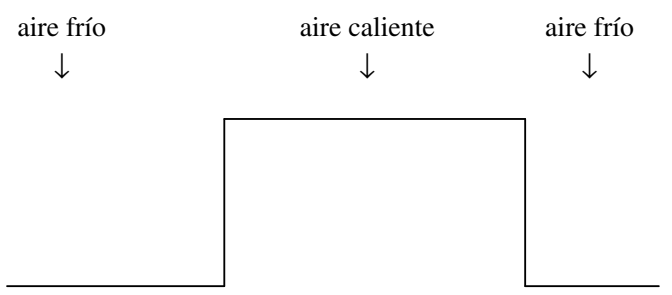

Pero además la qocha tiene otra aplicación en los Andes: preparar chuño; este consiste en papa deshidratada con las heladas, donde la qocha cumple una función de refrigeración y constituye una reserva alimenticia en el mundo andino.

El siguiente mapa permite ubicar la orografía y la hidrografía del área de referencia $\left(\right.$ Mapa 5) ${ }^{11}$.

Pero la crisis de la agricultura mencionada por Kolata afectó también el sistema agrícola de Moquegua que el autor califica como "colonias de Tiwanaku IV y V", ya que las lluvias alimentan la producción agrícola de la yunga, el autor sugiere que la crisis comenzó en los valles occidentales y arrastró al centro, convalidando así nuestro análisis de diferenciar el caso de Chucuito respecto del área de la denominada "Confederación aymara de la Provincia de Charcas".

El colapso de Tiwanaku implicó la reformulación del sistema, en el que Collas, Lupaqas y Pacajes tomaron la dirección de restablecer los controles sobre la yunga con los mitaqkuna, institución andina previa al período inca, durante este más bien fue utilizada con fines de expansión política. 


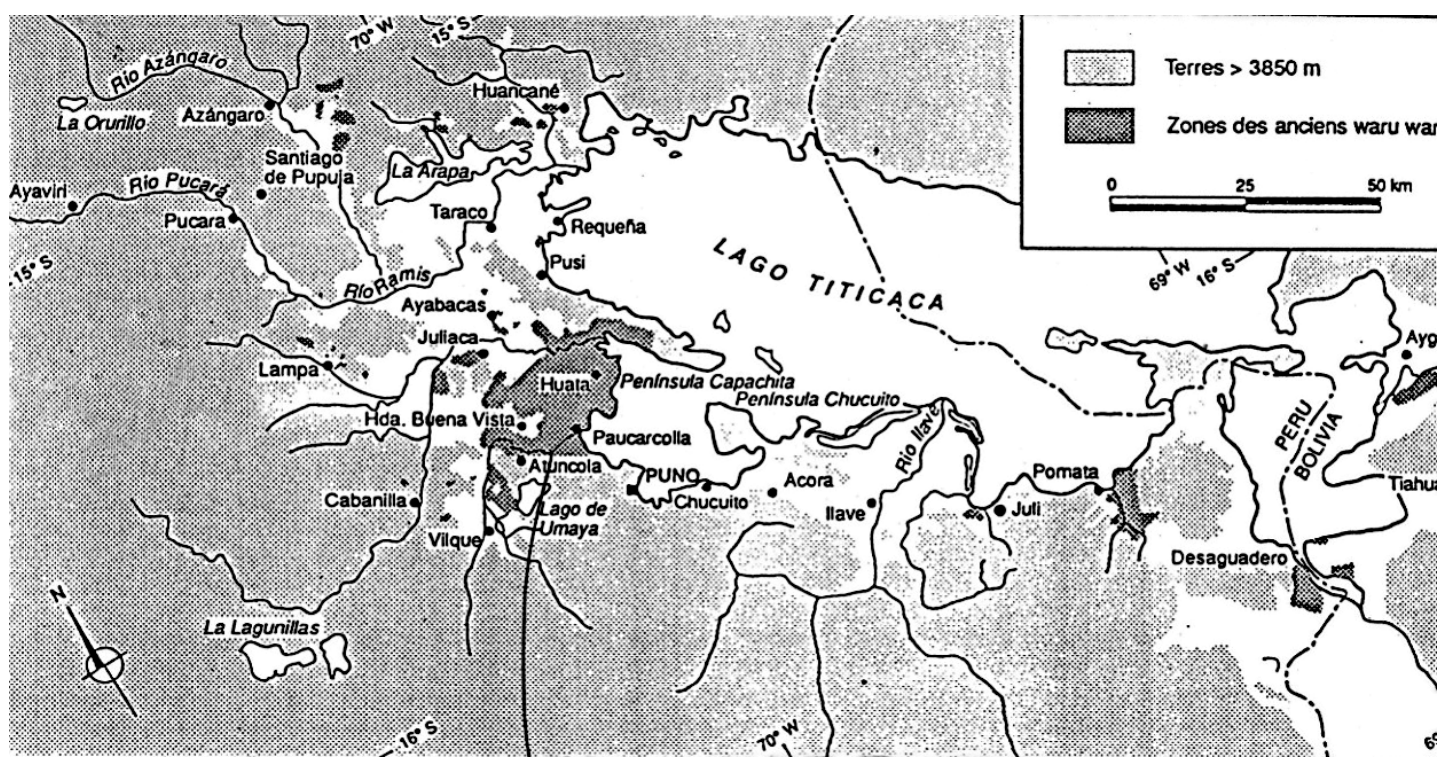

Mapa 5. Ver nota 11.

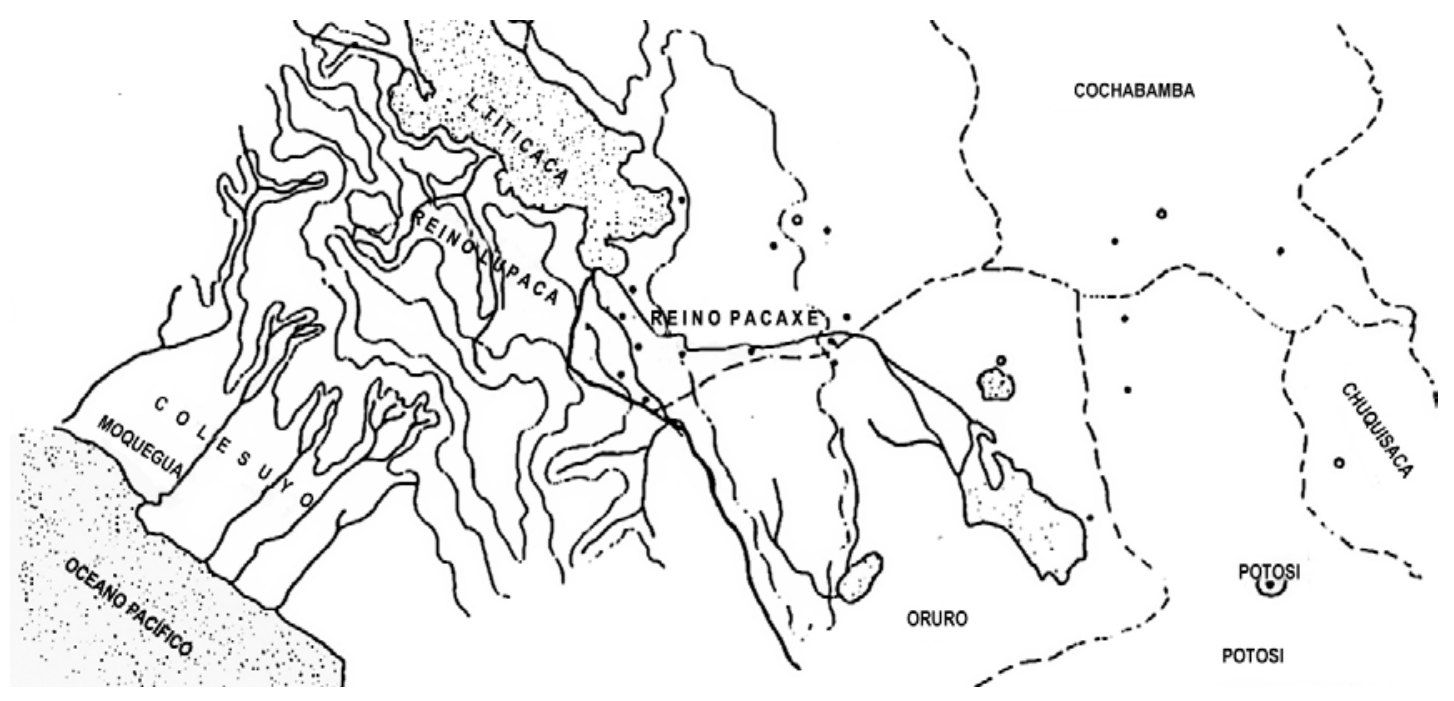

Mapa 6. Ver nota 12.

Asimismo, según la hipótesis de Kolata, implicó la primacía de la ganadería sobre la agricultura, de tal manera que la división entre Umasuyo y Urcusuyo que señala el Mapa $6^{12}$ es modificada por la ubicación del Colesuyo, que representa el área de articulación para los suministros agrícolas.

\section{Las articulaciones coloniales}

El mapa anterior nos señala el reino Lupaqa y la posición de los mitmaqkuna, que le permitió complementar recursos desde los distintos pisos ecológicos (Mapa 7) ${ }^{13}$. 


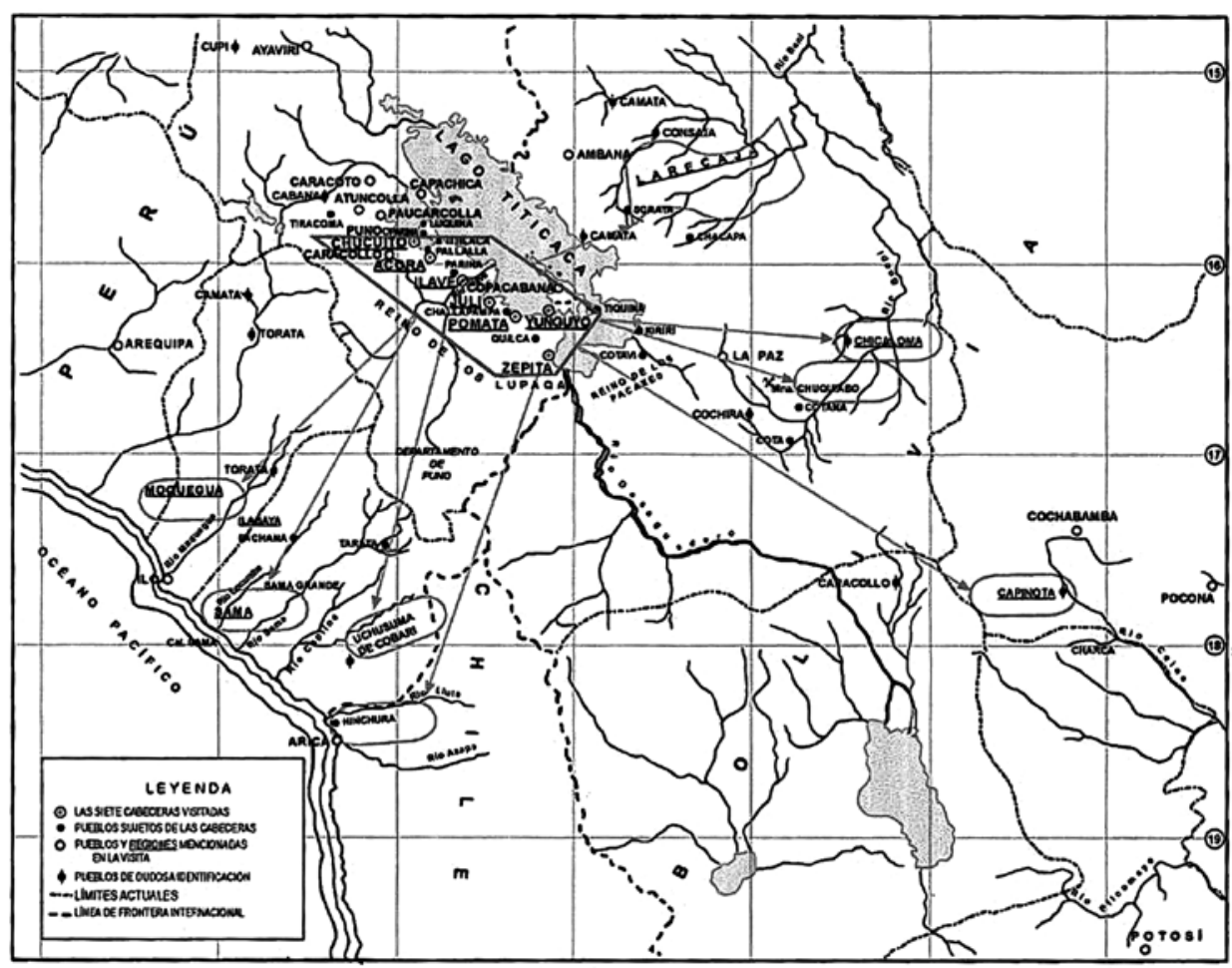

Mapa 7. Ver nota 13.

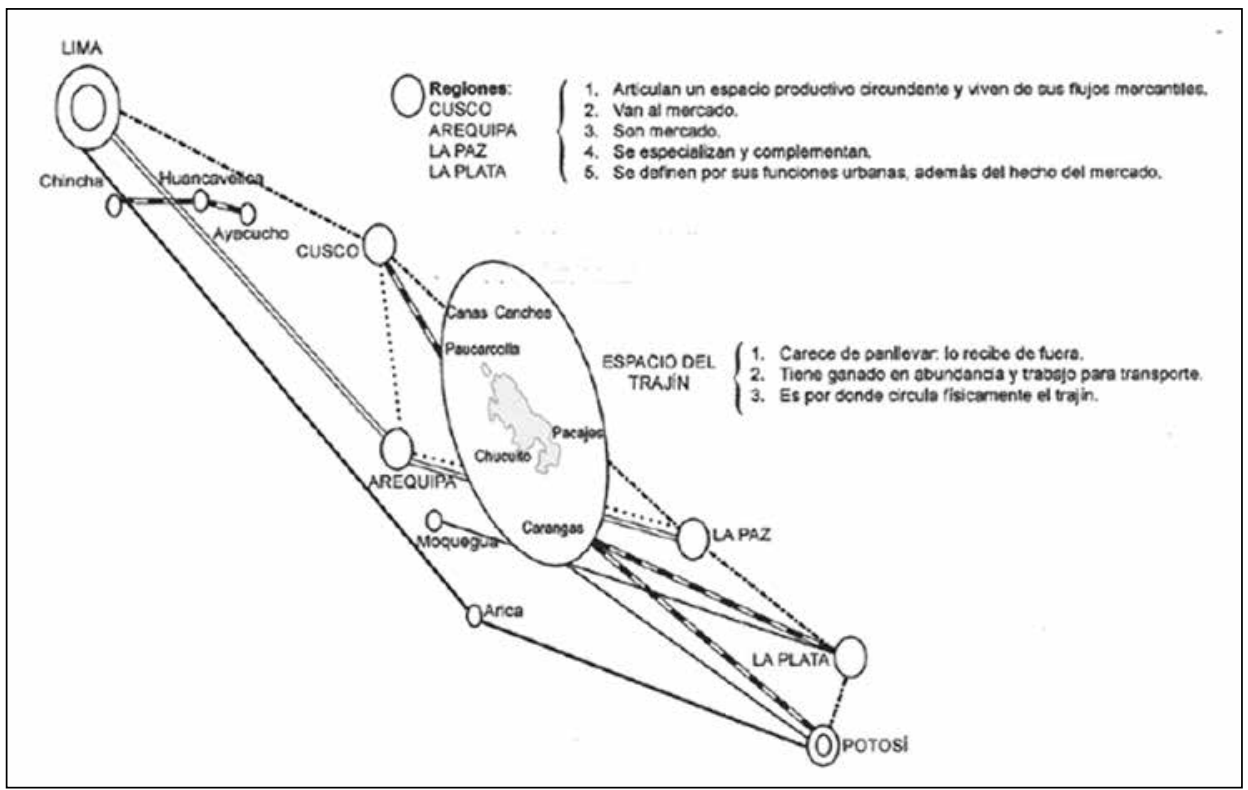

Esquema 1. Articulaciones prehispánicas.

Finalmente esas articulaciones prehispánicas fueron el antecedente del "espacio del trajín"14, como se aprecia a en el esquema 1.
Resulta claro que el espacio colonial se asentó sobre el área altiplánica objeto de esta presentación, la misma que se adaptó al nuevo circuito de tráfico mercantil. 


\section{Referencias Citadas}

Brouysse-Casagne, Thérèse

1978 "L'organisation de l'espace aymara", Annales, Economies, Société, Civilisations, 33: 5-6, Colin, Paris.

Brouysse-Cassagne, Thérèse Olivia Harris y TristanPlatt 2006 Qaraqara-Charka: Mallku, Inka y Rey en la provincia de Charcas (siglos XV-XVIII): historia antropológica de una confederación aymara. IFEA, Lima.

Cañedo-Argüelles Fábrega, Teresa

1993 Potosí: la versión aymara de un mito europeo: la minería y sus efectos en las sociedades andinas del siglo XVII (La provincia de Pacajes). Catriel, Madrid.

De la Torre, Carlos y Manuel Burga (Eds.)

1986 Andenes y camellones en el Perú andino: historia presente y futuro. CONCYTEC, Lima.

Glave, Luis Miguel

1989 Trajinantes: Caminos indígenas en la sociedad colonial, siglos XVI / XVII. Instituto de Apoyo Agrario, Lima.

Kolata, Alan Louis

1993 The Tiwanaku:portrait of an Andeancivilization. Blackwell, Cambridge.

Lumbreras, Luis Guillermo

1981 “El Imperio Wari”, En: Historia del Perú, II. Lima: Editorial Juan Mejia Baca.
Lemus Aguirre, Carlos

2006 "Reconfiguración sociopolítica y redes de tráfico e intercambio durante el período formativo en la cuenca del Titicaca”. En: Heather Lechtman (Ed.) Esferas de interacción prehistóricas y fronteras nacionales modernas: los Andes sur centrales. IEP, Lima e Institute of Andean Research, New York.

Saignes, Thierry

2007 Historia del pueblo chiriguano. IFEA, Lima

Salles, Estela Cristina y Noejovich, Héctor Omar

2008 "El reino Lupaqa y su área de influencia en el siglo XVI". En Salles y Noejovich, La visita general y el proyecto de gobernabilidad del virrey Toledo. UNSMP-Instituto de Gobierno, Lima

Salles, Estela Cristina y Noejovich, Héctor Omar

2013 "El repartimiento real de Chucuito en el virreinato del Perú: la tributación temprana y su evolución, 1539-1547", Fronteras de la Historia 18-2, ICAH, Bogotá.

Stanish, Charles, Amanda Cohen y Mark Aldenderfer (Eds.) 2006 Advances in Titicaca Basin Archeology. UCLA, Los Angeles.

Tarragó, Miriam Noemí

2006 En: Heather Lechtman (Ed.) Esferas de interacción prehistóricas y fronteras nacionales modernas: los Andes sur centrales. IEP, Lima e Institute of Andean Research, New York.

\section{Notas}

Fuente: Salles y Noejovich, 2013: 56.

2 Fuentes: Bouysse-Cassagne, Thérèse "L'organisation de l'espace aymara", Annales, Economies Société Civilisations, 33: 5-6. Paris: Colin, 1978; Stanish, Charles, Cohen Amanda y Mark Aldenderfer (Eds.) Advances in Titicaca Basinarchaeology. Los Angeles: UCLA, 2005. Lemus Aguirre, Carlos, "Reconfiguración sociopolítica y redes de tráfico e intercambio durante el período Formativo en la cuenca del lago Titicaca". En: Heather Lechtman, (Ed) Esferas de interacción prehistóricas y fronteras nacionales modernas: los Andes sur centrales. Lima: IEP; Nueva York: Institute of Andean Research, 2006.

3 Charles Stanish, Amanda Cohen y Mark S. Aldenderfert (Eds), ...op. cit., 2005.

4 Tarragó, Myriam Noemí “Espacios surandinos y la circulación de bienes en época del Tiawanaku". En: HeatherLechtman, (Ed) Esferas de interacción prehistóricas y fronteras nacionales modernas: los Andes sur centrales. Lima: IEP; Nueva York: Institute of Andean Research, 2006.

5 Luis Guillermo Lumbreras, 1981 "El Imperio Wari”, En: Historia del Perú, II. Lima: Editorial Juan Mejia Baca.

6 Thérèse Brouysse-Cassagne op. cit., 1992.

7 Como por ejemplo Thérèse Brouysse-Cassagne, Olivia Harris y Tristan Platt, Qaraqara-Charka: Mallku, Inka y Rey en la provincia de Charcas (siglos XV-XVIII): historia antropológica de una confederación aymara.Lima: IFEA
2006. Thierry Saignes, Historia del pueblo chiriguano. Lima: IFEA, 2007.

8 Alan Louis Kolata The Tiwanaku: portrait of an Andeancivilization. Cambridge: Blackwell 1993.

9 Cf. Carlos de la Torre y Manuel Burga (Eds.), Andenes y camellones en el Perú andino: historia presente y futuro. Lima: CONCYTEC 1986.

10 El Abra de La Raya, a 4300 m.s.m., es una línea divisora de las aguas, donde nace el río Vilcanota o Urubamba, afluente del Ucayali, cuyas aguas desembocan en el Atlántico; los ríos de la cuenca del Titicaca desembocan en el lago. También sería una suerte de "frontera étnica" entre quechuas y aymaras; como detalle simbólico indiquemos que actualmente es el límite entre los departamentos de Cuzco y Puno.

11 Fuente: Thérèse Brouysse-Cassagne, op. cit., 1992.

12 Fuente: Teresa Cañedo-Argüelles Fábrega, Potosí: la versión aymara de un mito europeo: la minería y sus efectos en las sociedades andinas del siglo XVII (La provincia de Pacajes). Madrid: Catriel, 1993.

13 Fuente: Estela Cristina Salles y Héctor Omar Noejovich, La visita y el proyecto de gobernabilidad del virrey Toledo. Lima: Universidad San Martín de Porras, 2008.

14 Luis Miguel Glave, Trajinantes: Caminos indígenas en la sociedad colonial, siglos XVI / XVII.Lima: Instituto de Apoyo Agrario, 1989. 
